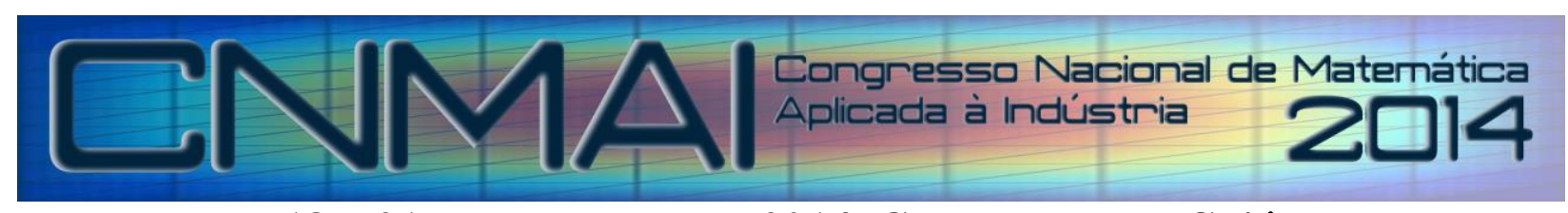

18 a 21 de novembro de 2014, Caldas Novas - Goiás

\title{
MELHORIA DE DESEMPENHO DE SISTEMAS DE CONTROLE DE VAZÃO EM SISTEMAS DE LIMPEZA CIP USANDO MODELOS SEMI- EMPÍRICOS DO TIPO FOPDT
}

\author{
Rubens Gedraite, rgedraite@feq.ufu.br ${ }^{1}$ \\ Fran Sérgio Lobato, fslobato@feq.ufu.br ${ }^{2}$ \\ Davi Leonardo de Souza, davilsouza@gmail.com ${ }^{3}$ \\ Leo Kunigk, kunigk@maua.br ${ }^{4}$
}

\author{
${ }^{1,2}$ UFU - Faculdade de Engenharia Química Av. João Naves de Ávila, 2121 - Uberlândia/MG \\ ${ }^{3}$ UFTM - Departamento de Engenharia Química Av.Randolfo Borges Jr., 1250, Uberaba/MG \\ ${ }^{4}$ CEUN-IMT - Coordenadoria de Pós-graduação Praça Mauá, 01 - São Caetano do Sul/SP
}

\begin{abstract}
Resumo:Atualmente, a grande maioria dos processos de limpeza CIP é conduzida com base em procedimentos que levam em consideração a experiência dos operadores de processo no que tange ao tempo de funcionamento do ciclo. Este trabalho aborda o comportamento do sistema CIP em resposta a estímulos na vazão de operação do sistema, usando um trocador de calor. A cinética da remoção foi avaliada com base na variação do valor do pH medido. A partir das respostas obtidas, foram identificados os modelos do tipo FOPDT representativos da dinâmica do processo estudado para diferentes condições operacionais, que foram validados por meio de comparação com os dados experimentais disponíveis, sugerindo representatividade adequada e coerente. Os modelos foram usados para estudo da influência da oscilação nos valores da vazão de água de enxágue, em torno do ponto de operação, sobre o $\mathrm{pH}$. A partir dos desvios avaliados foi feito o ajuste dos parâmetros do controlador de vazão de água de enxágue, visando a diminuição da variabilidade apresentada. O desvio máximo verificado foi inferior a 5\%.
\end{abstract}

Palavras-chave: sistema de limpeza CIP,cinética de remoção de resíduos, modelo semi-empírico, controle de vazão

\section{INTRODUÇÃ̃}

A quantidade de água gasta nos processos de limpeza, em especial nos processos CIP, vem aumentando muito a cada ano, em decorrência da intensificação da produção de alimentos. Por se tratar de tema definido pelos órgãos de vigilância sanitária, não são poupados esforços para assegurar que o processo de limpeza dos equipamentos seja adequado e aderente aos critérios estabelecidos na legislação em vigor. Entretanto, este fato tradicionalmente implica na utilização, por parte da comunidade industrial, de maior quantidade de insumos do que aqueles que seriam necessários e tecnicamente suficientes para atender aos requisitos técnicos estabelecidos nas leis aplicáveis (Bansal; Chen, 2006).

Por serem procedimentos que requerem paradas de produção, os processos de higienização, muitas vezes, são realizados de forma negligenciada pelas empresas. Portanto, é de fundamental importância que sejam estudados e otimizados, através do estabelecimento das cinéticas de remoção de resíduos de cada etapa do processo (Gedraite et al., 2013).

O estabelecimento do tempo adequado ao processo de higienização é fundamental para a eficiência do processo. Deve ser suficientemente longo para que as reações químicas e as interações físicas ocorram a contento; mas não deve ser excessivo, pois reduziria a produtividade da indústria (Carlotti, 2008).

Um processo de higienização é composto pelas seguintes etapas: pré-lavagem, circulação de solução detergente, enxágue e circulação de solução com ação antimicrobiana (sanificante). As etapas estudadas serão: aplicação de solução do detergente e o enxágue. A primeira consiste na ação do detergente sobre os resíduos para que estes sejam removidos da superfície dos equipamentos. O enxágue se caracteriza pela passagem de água no equipamento com função de remoção do detergente químico utilizado e dos resíduos deslocados pelo mesmo. 
Dois parâmetros são tipicamente envolvidos em processos desta natureza, a saber: vazão de escoamento e temperatura. O primeiro está relacionado com a ação cisalhante proporcionada pela solução de detergente e água de enxágue sobre a superfície, enquanto o segundo permite melhor solubilização dos resíduos, facilitando sua remoção. Em um sistema de limpeza CIP, o tempo de escoamento das soluções, em cada etapa do processo de higienização, é o parâmetro de maior facilidade de manipulação e, por isso, o fator preferido de redução. Porém, se alterado de forma não criteriosa pode ocasionar a não efetividade do processo (Gormezano, 2007).

O equipamento estudado para este trabalho foi um trocador de calor do tipo casco e tubos, de difícil higienização, pois as temperaturas envolvidas durante seu funcionamento acarretam, entre outros, na desnaturação proteica que dificulta a retirada dos resíduos, obrigando a realização de paradas mais frequentes (Gedraite et al., 2011).

A prática de higienização de equipamentos pela indústria de alimentos baseia-se em experiências empíricas, deixando o setor à mercê de informações provenientes dos fornecedores de detergentes e sanitizantes. Parâmetros semiempíricos são adotados a partir de resultados finais de higienização considerados satisfatórios. A complexidade dos fenômenos existentes nas reações de remoção requer o aumento de trabalhos científicos que possam contribuir com os procedimentos adotados industrialmente (Oliveira et al., 2012).

\section{MATERIAIS E MÉTODOS}

O sistema utilizado para os testes experimentais desenvolvidos por Melero Jr. (2011) é apresentado no fluxograma da Figura 1. Ele é composto basicamente pelos seguintes elementos: (i)- tanque principal em aço inox com capacidade para $250 \mathrm{~L}$, (ii)- trocador de calor do tipo casco e tubos em aço inox, (iii)- bomba de deslocamento positivo para movimentação do produto alimentício, (iv)- bomba centrífuga para a movimentação do agente de aquecimento e (v)reservatório cilíndrico vertical para armazenamento da água de aquecimento.

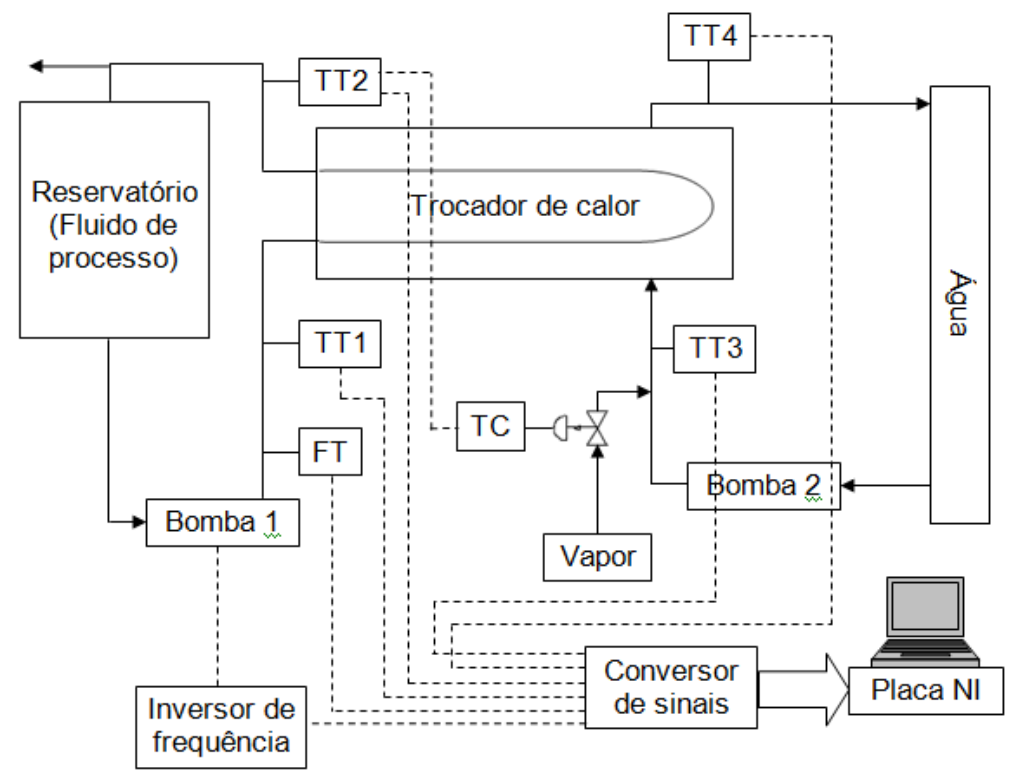

Figura 1. Fluxograma de processo e instrumentação do sistema estudado.

Melero Jr. (2011) submeteu o trocador de calor à operação com leite e posteriormente efetuou a limpeza do mesmo por meio do emprego de solução de hidróxido de sódio a $0,5 \%$ em massa, o qual atua como detergente neste processo. Na sequência, o trocador de calor foi lavado com água de enxágue para remoção dos resíduos de soda caustica.

A medição da concentração residual de hidróxido de sódio presente na água de enxágue usada foi medida experimentalmente por meio de um medidor de $\mathrm{pH}$ de laboratório, adaptado para medição em tempo real. As medições das temperaturas terminais do trocador de calor foi realizada por meio de elementos sensores de temperatura do tipo resistência de Platina (Pt 100) e a medição da vazão foi realizada por meio de medidor de vazão de engrenagens ovais.

Os resultados obtidos com a medição do $\mathrm{pH}$, das temperaturas terminais do trocador de calor e da vazão de produto ao longo do tempo foram coletados eletronicamente por meio de um sistema de aquisição de dados utilizando o aplicativo Labview®.

A Figura 2 mostra a facilidade experimental utilizada por Melero Jr (2011). O funcionamento do trocador de calor (P1) consiste no escoamento do fluído de processo, alimentado no estado líquido, feita a partir do tanque intermediário (P5). O fluido de processo é introduzido no lado dos tubos do trocador de calor, conforme apresentado na Figura 3. 


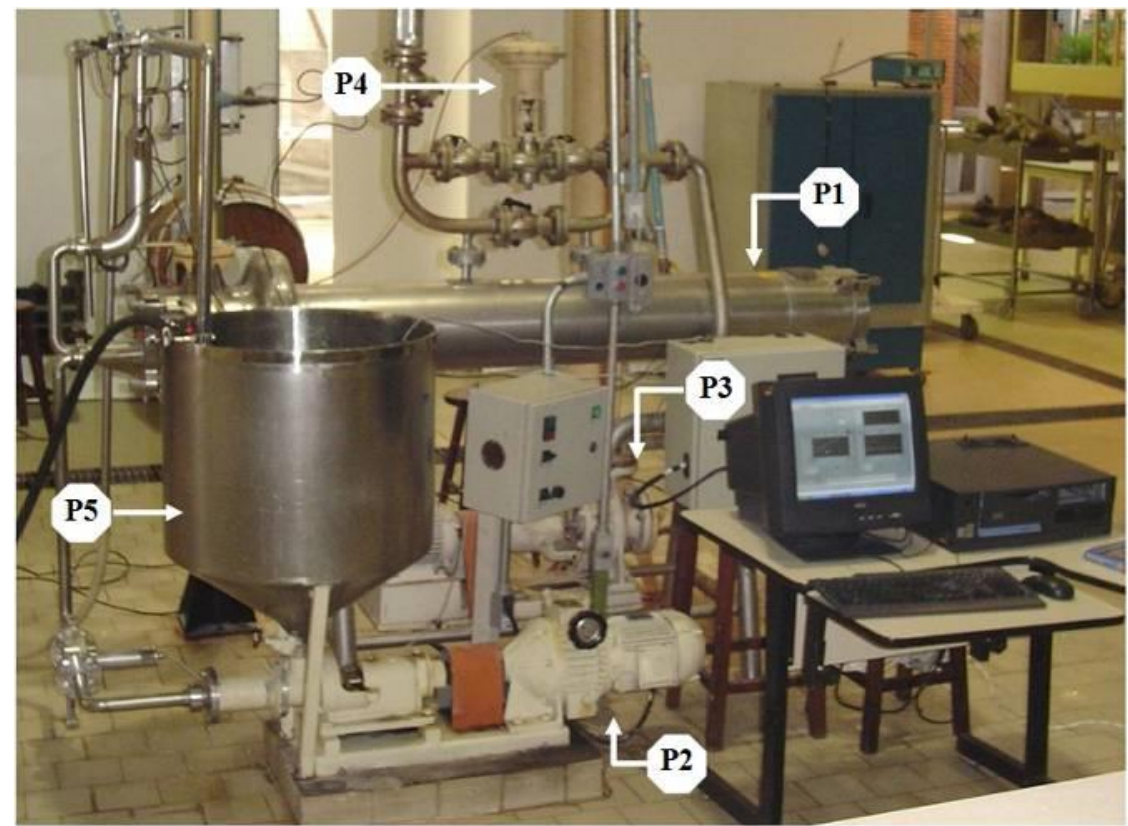

Figura. 2 Trocador de calor tipo feixe tubular estudado.

O feixe tubular contempla 4 passagens pelo lado dos tubos, conforme esquematizado na Figura 4. Nos tubos, o fluído de processo escoa inicialmente pela seção 1, em seguida segue pela seção 2 e assim sucessivamente pelas seções 3 e 4. Por meio do emprego de válvulas manuais, o fluxo do fluído de processo na saída do feixe tubular do trocador de calor pode ser desviado para o reservatório de entrada ou para duas saídas de descarte.

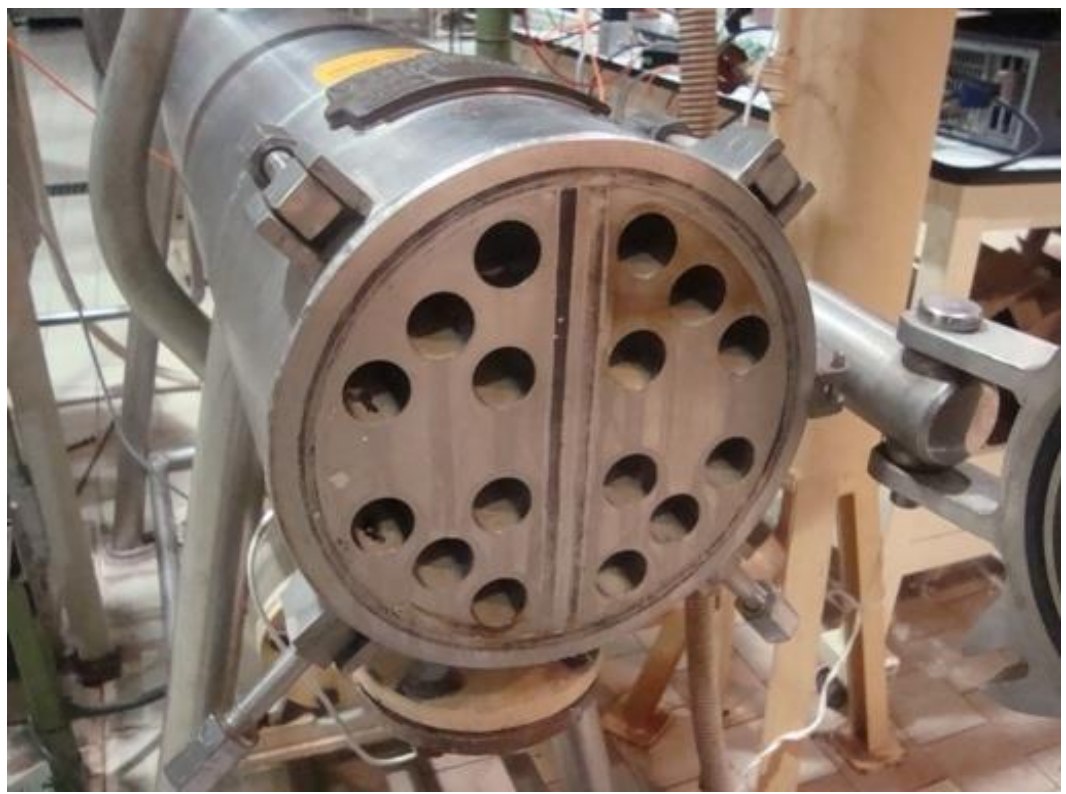

Figura 3. Espelho do trocador de calor estudado.

O deslocamento do fluído de processo nos tubos internos do trocador de calor é promovido por uma bomba de deslocamento positivo (P2) mostrada na Figura 2. A vazão do fluido através da bomba é ajustada por meio de um inversor de frequência. 


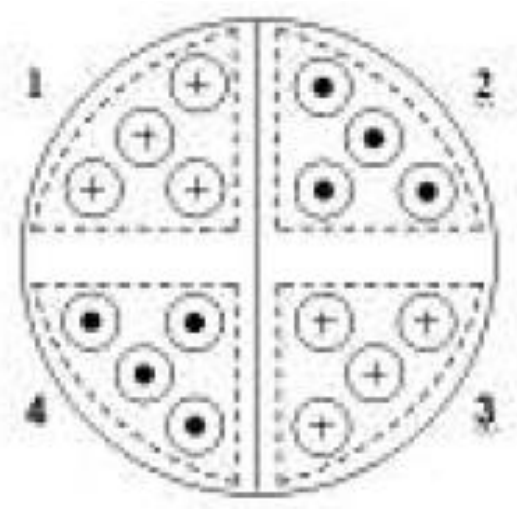

Figura 4. Representação do número de passagens do fluido pelo lado dos tubos.

O aquecimento do trocador de calor, é realizado com água quente que circula por sua carcaça. A água de aquecimento é aquecida por meio de vapor de água saturado, alimentado diretamente no circuito fechado de aquecimento. A água quente é feita escoar pela bomba centrífuga (P3) mostrada na Figura 2, com acionamento tipo liga/desliga.

O controle da temperatura de saída do fluido de processo, do lado dos tubos, é feito por meio do ajuste da vazão de vapor de aquecimento, utilizando-se a válvula de controle pneumática (P4). Na Figura 5 é apresentada a válvula de controle utilizada.

Melero Jr. (2011) realizou ensaios para a obtenção dos parâmetros do processo referentes à resposta da planta à alterações na temperatura de operação do equipamento.

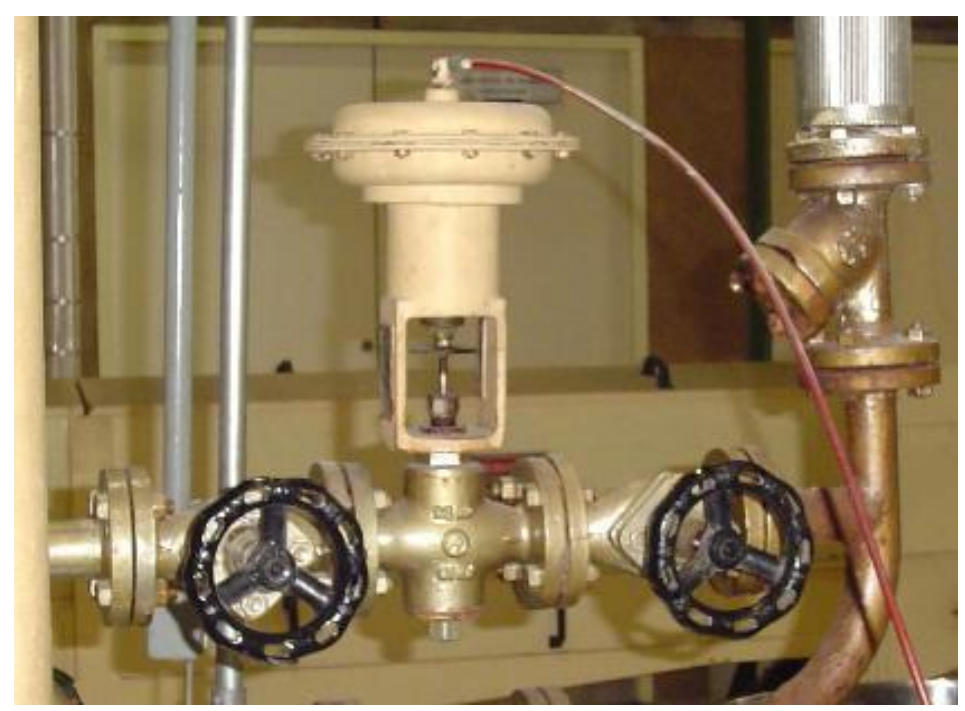

Figura 5. Válvula de controle pneumática.

No ensaio realizado, Melero Jr. (2011) utilizou a temperatura constante de $50{ }^{\circ} \mathrm{C}$. Partiu-se da condição em que a vazão da água de enxágue era igual a 4,0 L/min, aplicou-se uma variação brusca do tipo degrau no sinal de saída do controlador de vazão - em modo manual - o que provocou o aumento do sinal de controle enviado ao inversor de frequência. As análises para obtenção dos parâmetros do processo foram realizadas tomando-se por base o degrau aplicado.

O modelo da planta em estudo foi desenvolvido de maneira a contemplar o cenário de flutuações no valor da vazão de água de enxágue, que tipicamente são verificadas nas instalações industriais. Neste contexto, o modelo levou em conta o ajuste dos valores dos parâmetros de acordo com a vazão empregada. Na Figura 6 é apresentado, a título de ilustração, o diagrama de simulação típico, considerando o modelo de referencia identificado e o modelo do processo em estudo. Os valores dos parâmetros $K_{P}, \tau_{P}$ e $\theta_{p}$ foram obtidos com base nos experimentos realizados.

As flutuações no valor da vazão foram modeladas por meio de variação aleatória das mesmas em torno do valor em ajustado, segundo uma distribuição normal, com comportamento randômico, com média igual a zero e variância igual a $1,0 \mathrm{~L} / \mathrm{min}$. $\mathrm{O}$ valor assumido para a variância corresponde àquele tipicamente verificado em malhas de controle de vazão de acordo com a precisão dos elementos que a compõem. O sinal gerado pelo bloco de função responsável por produzir a flutuação do valor da vazão é somado ao sinal de vazão estudado, como mostrado na Figura 6. 


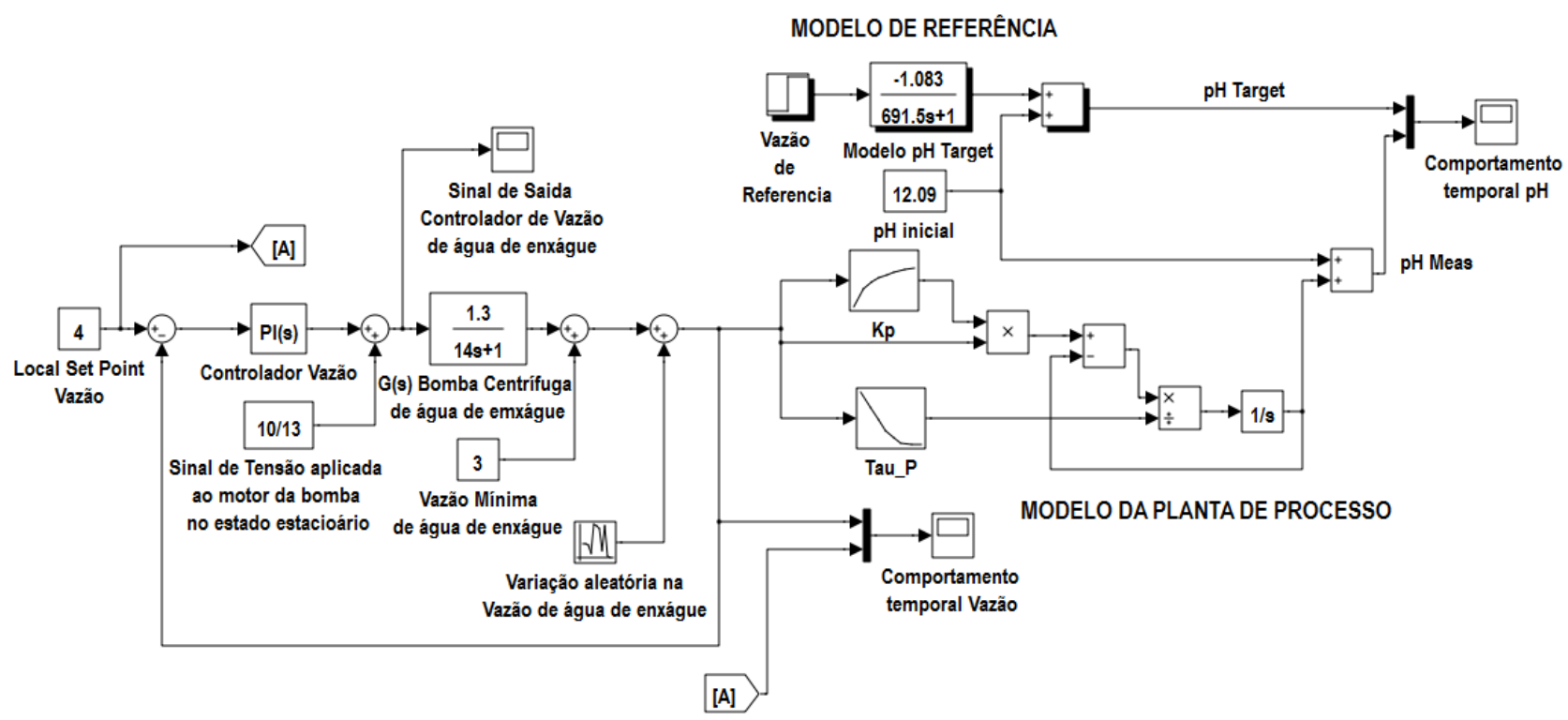

Figura 6. Diagrama de simulação típico do processo em estudo.

\section{RESULTADOS OBTIDOS \& DISCUSSÃO}

Os parâmetros do processo relacionado com o estudo da dinâmica da vazão, $K_{P}$ (ganho), $\tau_{P}$ (constante de tempo) e $\theta_{P}$ (atraso de transporte), foram calculados segundo o método proposto por Smith (1972) e por Garcia (2005). A função de transferência obtida é apresentada na Equação (1).

$$
G(s)=\frac{1,3 \cdot e^{-2 \cdot s}}{14 \cdot s+1}
$$

Utilizando os parâmetros do processo obtidos $\left(K_{P}, \tau_{P}\right.$ e $\left.\theta_{P}\right)$, foram empregados vários métodos para sintonia de controladores propostos em Campos e Teixeira (2007), sendo eles: ZN - Ziegler \& Nichols, CC - Cohen \& Coon e ITAE - Integral do Módulo do Erro vezes o Tempo por Lopez (Miller et al, 1967). Os parâmetros de sintonia do controlador de vazão com modos de controle $P I$ foram calculados e são apresentados na Tabela 1 . Foi considerado que $K_{C}$ é o ganho proporcional e $T_{I}$ o tempo integral.

O critério utilizado para escolher os valores mais adequados dos parâmetros de sintonia foi o ISE - Integrated Square Error. Neste critério, o desvio entre o valor medido e o set-point é elevado ao quadrado e integrado ao longo do tempo (Mcmillan, 1994).

Tabela 1 - Valores dos parâmetros de sintonia do controlador de vazão.

\begin{tabular}{ccc}
\hline Método & $K_{C}$ & $T_{I}$ \\
\hline \hline ZN & 4,85 & 0,111 \\
CC & 4,91 & 0,022 \\
ITAE-Lopez & 2,68 & 0,164 \\
\hline
\end{tabular}

A partir dos parâmetros de sintonia ajustados para o controlador de vazão considerado, foram executadas simulações computacionais, usando o aplicativo Matlab/Simulink®. Os valores de ISE calculados são apresentados na Tabela 2.

Tabela 2 - Valores calculados para ISE.

\begin{tabular}{cc}
\hline Método & $I S E$ \\
\hline \hline ZN & $-1,476 \mathrm{E} 77$ \\
CC & $9.46 \mathrm{E} 155$ \\
ITAE-Lopez & $1,682 \mathrm{E}-9$ \\
\hline
\end{tabular}

O melhor ajuste para os parâmetros de sintonia do controlador de vazão foi obtido com o emprego do método ITAE-Lopez, o qual apresentou valores de parâmetros de sintonia que sugerem um comportamento de correção mais 
suave e o menor valor de ISE. Os valores de $K_{C}$ calculados pelos métodos de ZN e de CC são significativamente próximos entre si, enquanto que aquele calculado por ITAE-Lopez é bastante próximo dos anteriores. O valor de $T_{I}$ calculado pelo método de $\mathrm{CC}$ é o dobro daquele calculado por $\mathrm{ZN}$, enquanto que aquele calculado por ITAE-Lopez é aproximadamente $50 \%$ menor do que o calculado por $\mathrm{ZN}$ e muito próximo daquele calculado por CC. Valores de $T_{I}$ menores tendem a contribuir de maneira mais efetiva para o retorno da variável controlada ao set-point.

Com base nos resultados experimentais obtidos, foram utilizados no modelo matemático aproximado da planta, do tipo FOPDT, os parâmetros de sintonia do controlador de vazão visando avaliar o comportamento dinâmico da cinética de remoção de resíduos em função da vazão considerada na etapa de enxágue. Cumpre ressaltar que os valores dos parâmetros identificados do modelo da planta estudada são apresentados na Tabela 3 (Gedraite et al., 2011).

Nas Figuras 7, e 8 são apresentados os comportamentos temporais da variação aleatória imposta à vazão de água de enxágue e da vazão controlada de água de enxágue.resultados das simulações realizadas no aplicativo Matlab/Simulink®, para as condições postuladas, utilizando o modelo de referencia ( $p H$ target) e o modelo da planta de processo ( $p H$ meas).

Tabela 3. Parâmetros dos modelos matemáticos FOPDT do processo estudado.

\begin{tabular}{ccc}
\hline $\begin{array}{c}\text { Degrau } \\
{[\boldsymbol{L} / \boldsymbol{m i n}]}\end{array}$ & $\begin{array}{c}\text { Ganho, } \boldsymbol{K}_{\boldsymbol{p}} \\
{[\boldsymbol{p H} /(\boldsymbol{L} / / \mathbf{m i n})]}\end{array}$ & $\begin{array}{c}\text { Constante de tempo, } \tau_{\boldsymbol{p}} \\
{[\boldsymbol{s}]}\end{array}$ \\
\hline 4,0 & $-1,083$ & 691,5 \\
\hline
\end{tabular}

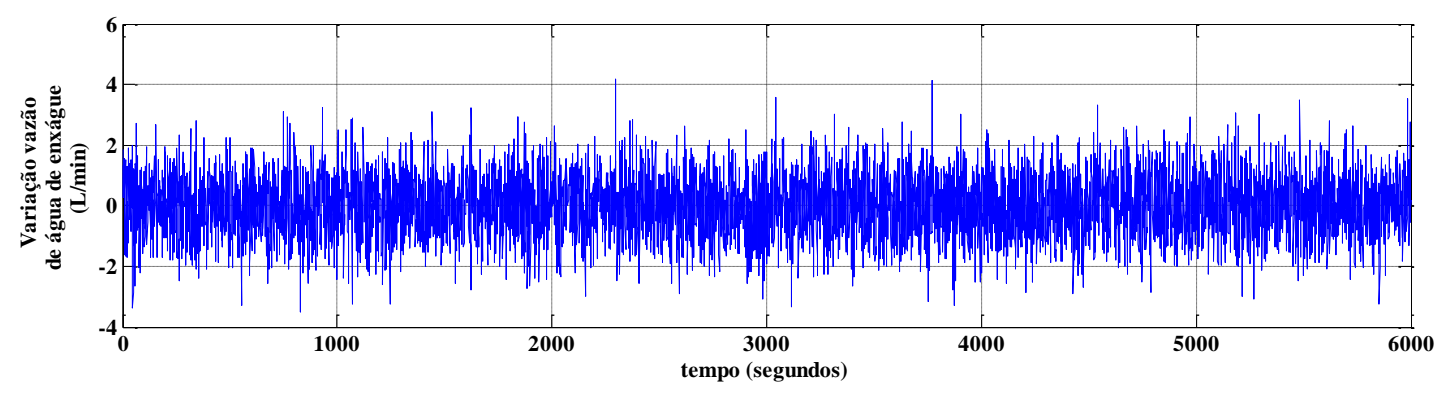

Figura 7. Comportamento temporal da variação aleatória imposta à vazão de água de enxágue.

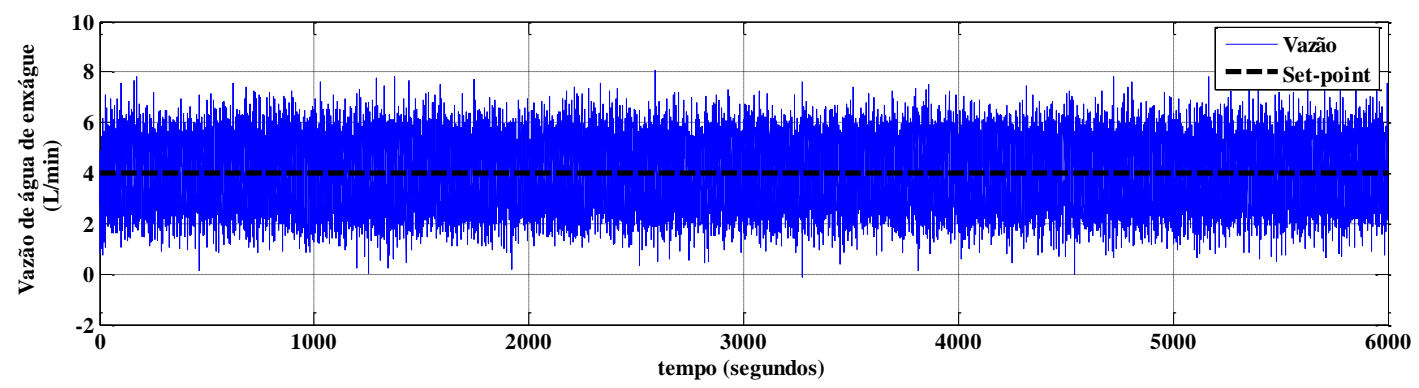

Figura 8. Comportamento temporal da vazão de água de enxágue.

Na Figura 9 é apresentado o comportamento temporal da variação do $p H$ meas e do $p H$ target para a vazão de $4 \mathrm{~L} / \mathrm{min}$, obtidos com base nas simulações realizadas no aplicativo Matlab/Simulink®.

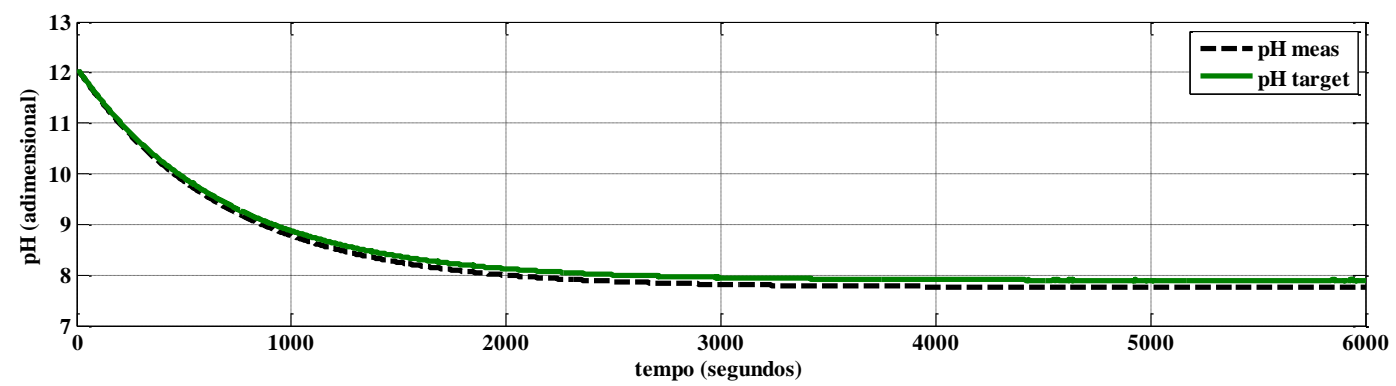

Figura 9. Comportamento temporal do $\mathrm{pH}$. 
Com base nos resultados obtidos, pode-se constatar que a influência das perturbações impostas no valor da vazão foi pouco significativa sobre o comportamento temporal da variação do $\mathrm{pH}$ meas, com desvio padrão aproximadamente igual a 0,9144 , em que pese o fato da amplitude escolhida para as perturbações $(1 \mathrm{~L} / \mathrm{min})$ representar aproximadamente $25 \%$ do valor de vazão usada no experimento.

\section{CONCLUSÕES}

Os modelos matemáticos encontrados são bastante simples e adequados para fácil implementação na base de dados de um sistema digital de controle tipicamente utilizado na indústria alimentícia, visando realizar o acompanhamento em tempo real da operação da etapa de enxágue do processo de limpeza pelo sistema CIP.

Diante dos resultados apresentados, pode-se concluir que o modelo identificado da planta revela boa aderência com as cinéticas de remoção de resíduos estudadas, podendo ser usado como ferramenta de analise e/ou projeto de processo. Com base no modelo, pode-se avaliar o impacto que alterações pré-estabelecidas no valor da vazão de água de enxague podem apresentar sobre o tempo de operação e também sobre o volume total de água gasto em cada processo.

Deve ser ressaltado que, como os modelos anteriormente descritos são semi-empíricos, toda sistemática adotada neste trabalho é válida para inferência das mesmas grandezas nas outras etapas envolvidas em processos de limpeza pelo sistema CIP.

Os parâmetros de sintonia ajustados para o controlador de vazão sugerem bom desempenho da variável de processo $p H$ meas, considerada como indicador chave de performance para o sistema em estudo. Com base nos resultados obtidos, pode-se verificar que o emprego de modelos semi-empíricos para análise e acompanhamento do processo de limpeza considerado neste trabalho sugere a possibilidade de redução no tempo de operação do processo e, por consequência, de redução no consumo de energia e de água de enxágue através do cálculo remoto e em tempo real do valor do set-point do controlador de vazão, ao invés de se utilizar um valor fixo.

\section{AGRADECIMENTOS}

Os autores agradecem à UFU, ao IMT e à Fapemig (Fundação de Amparo à Pesquisa do Estado de Minas Gerais) pelo apoio em pesquisas no projeto No TEC-APQ-02100-12 (Estudo da Otimização de Sistema de Limpeza CIP).

\section{REFERÊNCIAS}

Aguirre, L. A., 2007. Introdução à identificação de sistemas: técnicas lineares e não lineares aplicadas a sistemas reais. Ed. UFMG.

Bansal, B.; Chen, X. D. 2006. A critical review of milk fouling in heat exchangers. Comprehensive reviews in food science and food safety, vol. 5, $\mathrm{n}^{\circ} .2$, pp. 27-33.

Campos, M. C. M. M; Teixeira, H. C. G. 2007. Controles Típicos de Equipamentos e Processos Industriais. Ed. Edgard Blücher, São Paulo.

Carlotti Filho, M. A. C. 2008. Estudo da cinética de remoção de resíduos de cálcio e de detergente alcalino nas etapas de pré-lavagem e de enxágue em um trocador de calor de placas. Dissertação de mestrado, CEUN-IMT.

Chen, X. D.; Ozkany, N.; Qin, F.; Xin, H.; Lin, L. 2004. An effective CIP procedure for removing dairy protein based deposit - A Laboratory investigation. ECI Conference on heat exchanger fouling and cleaning fundamentals and applications, Santa Fe, New Mexico, paper 44, 07p.

Garcia, C. 2005. Modelagem e Simulação de Processos Industriais e de Sistemas Eletromecânicos. EDUSP, São Paulo $-\mathrm{SP}, 678 \mathrm{p}$.

Gedraite, R.; Kunigk, L.; Ribeiro, S.; Melero Jr., V.; Vasconcelos, F.; Sislian, R. 2010. Experimental investigation about the milk protein based deposit removal kinetics. Proceedings of the World congress on communication and arts, WCCA, Guimarães - Portugal.

Gedraite, R., Lobato, F. S., Neiro, S. M. S., Melero Jr., V., Augusto, S. R., Kunigk, L. 2011. Modelagem matemática da cinética de remoção de resíduos em sistemas de limpeza CIP. Proceedings of the XXXII Iberian Latin-American Congress on Computational Methods in Engineering. Rio de Janeiro, Brazil.

Gedraite, R., Pinheiro, T. M. A., Coutinho Filho, U. Kunigk, L, e Neiro, S. M. S. Investigação da limpeza de depósitos proteicos na superfície de aço inox 316 por processo CIP com hidróxido de sódio. Anais do VII Congresso Brasileiro de Termodinâmica Aplicada (CBTermo - 2013). Uberlândia, Brasil.

Gormezano, L. 2007. Desenvolvimento e implementação de sistema para avaliar a cinética de remoção de resíduos presentes nos tubos de trocador de calor feixe tubular. Dissertação de mestrado, CEUN-IMT.

Mcmillan, G. K. Tuning and control loop performance. $3^{\text {rd }}$ ed., Research Triangle Park, NC, ISA - The Instrumentation, Systems and Automation Society, 1994.

Melero Jr., V. 2011. 'Instrumentação e identificação de um processo de sanitização cinética CIP'. Centro Universitário do Instituto Mauá de Tecnologia, São Caetano do Sul - (Dissertação de Mestrado).

Miller, J. R. Miller, J. A.; Lopez, A. M.; Smith, C. L.; Murrill, P. W. 1967. A comparison of controller tuning techniques. Control Engineering, vol. 14, nº 12, pp. 72.

Smith, C. L. 1972. Modeling and simulation in the process industries. National computer conference and exposition Publisher. Department of Chemical Engineering. Lousiana State University, Baton Rouge. Lousiana. 
Oliveira, L. R., Gedraite, R.; Coutinho Filho, U. \& Kunigk, L. 2012. Obtenção de modelo empírico para avaliação da quantidade de insumos gastos em um sistema CIP empregado na limpeza de um sistema de pasteurização de leite. Anais do XV Encontro de Modelagem Computacional e III Encontro de Ciência e Tecnologia de Materiais. Uberlândia, Brasil.

Salvagnini, W.; Gedraite, R. (2001). Rotina de Experimento para trocador de calor feixe tubular. São Caetano do Sul, SP: CEUN-IMT.

\title{
RESPONSABILIDADE AUTORAL
}

Os autores são os únicos responsáveis pelo conteúdo deste trabalho.

\section{CIP SYSTEMS FLOWRATE CONTROL IMPROVEMENT APPLYING FOPDT MODELS}

\author{
Rubens Gedraite, rgedraite@ feq.ufu.br ${ }^{1}$ \\ Fran Sérgio Lobato, fslobato@feq.ufu.br ${ }^{2}$ \\ Davi Leonardo de Souza, davilsouza@gmail.com ${ }^{3}$ \\ Leo Kunigk, kunigk@maua.br ${ }^{4}$
}

\author{
${ }^{1,2}$ UFU - Faculdade de Engenharia Química Av. João Naves de Ávila, 2121 - Uberlândia/MG \\ ${ }^{3}$ UFTM - Departamento de Engenharia Química Av.Randolfo Borges Jr., 1250, Uberaba/MG \\ ${ }^{4}$ CEUN-IMT - Coordenadoria de Pós-graduação Praça Mauá, 01 - São Caetano do Sul/SP
}

\begin{abstract}
Currently, the vast majority of CIP processes is conducted based on procedures that take into account the experience of process operators with respect to the operating time of the cycle. This paper discusses the behavior of the CIP system in response to system flow changes, using a shell and tube heat exchanger. The kinetics of removal was evaluated based on the change in the value of the measured pH. From the responses obtained, FOPDT models representative of the process dynamics were studied for different operating conditions, which were validated by comparison with experimental data available, suggesting appropriate and consistent representation. The models were used to study the influence of rinse water flow oscillation, around the operating point on the $p H$. The deviations were evaluated and flow controller tunnig parameters adjusted, aiming to reduce the variability shown. The maximum deviation observed was less than 5\%.
\end{abstract}

Keywords: CIP system,waste removal kinectics, FOPDT model, flow control 